PROCEEDINGS OF THE

AMERICAN MATHEMATICAL SOCIETY

Volume 127, Number 1, January 1999, Pages 259-264

S 0002-9939(99)04490-1

\title{
ON THE TOPOLOGY OF ISOPARAMETRIC HYPERSURFACES WITH FOUR DISTINCT PRINCIPAL CURVATURES
}

\author{
FUQUAN FANG
}

(Communicated by Christopher Croke)

\begin{abstract}
Let $\left(m_{-}, m_{+}\right)$be the pair of multiplicities of an isoparametric hypersurface in the unit sphere $S^{n+1}$ with four distinct principal curvatures -w.r.g., we assume that $m_{-} \leq m_{+}$. In the present paper we prove that, in the case $4 \mathrm{~B} 2$ of U. Abresch (Math. Ann. 264 (1983), 283-302) (i.e., where $\left.3 m_{-}=2\left(m_{+}+1\right)\right), m_{-}$must be either 2 or 4 .

As a by-product, we prove that the focal manifold $F_{-}$of an isoparametric hypersurface is homeomorphic to a $S^{m_{+}}$bundle over $S^{m_{+}+m_{-}}$if one of the following conditions holds: (1) $m_{+}>m_{-}>1$ and $m_{+}=3,5,6$ or $7(\bmod 8)$; (2) $m_{+}>2 m_{-}>2$ and $m_{+}=0(\bmod 4)$. This generalizes partial results of Wang (1988) about the topology of Clifford type examples. Consequently, the hypersurface is homeomorphic to an iterated sphere bundle under the above condition.
\end{abstract}

\section{INTRODUCTION}

A hypersurface in the unit sphere $S^{n+1}$ is called isoparametric if it has constant principal curvatures. E. Cartan [4] first considered the cases of isoparametric hypersurfaces with 1, 2 or 3 distinct principal curvatures and solved the classification problem. Münzner [7] proved that the number $g$ of distinct principal curvatures of an isoparametric hypersurface in $S^{n+1}$ is $1,2,3,4$ or 6 . Some restrictions about the multiplicities in the latter two cases were treated in [1]. By [1], the dimension $n$ has to be 6 or 12 when $g=6$.

The situation when $g=4$ is much more complicated and interesting. By using Clifford algebra, infinite families of isoparametric hypersurfaces in $S^{n+1}$ with four distinct principal curvatures were constructed by Ferus, Karcher and Münzner. The topology of those examples was studied in [10]. Some very interesting necessary conditions about the multiplicities of $\frac{g}{2}$ curvatures, $m_{+}$and $m_{-}$, have been obtained by Abresch [1]. Notice the well-known formula $2 n=g\left(m_{+}+m_{-}\right)$. In order to remind the reader, we recall the following

Theorem (Abresch [1]). Assume given an isoparametric hypersurface in $S^{n+1}$ with $g=4$. Then the pair $\left(m_{+}, m_{-}\right)$-w.r.g. we may assume that $m_{-} \leq m_{+}-$satisfies one of the following conditions:

Received by the editors June 21, 1995 and, in revised form, February 1, 1996 and April 30, 1997.

1991 Mathematics Subject Classification. Primary 53C40; Secondary 53B25.

Key words and phrases. Isoparametric hypersurface, principal curvature, multiplicity of principal curvature, iterated sphere bundle.

(C)1999 American Mathematical Society 
4A. $m_{+}+m_{-}+1$ is divisible by $2^{k}:=\min \left\{2^{\sigma} \mid 2^{\sigma}>m_{-}, \sigma \in N\right\}$.

4B1. $m_{-}$is a power of 2 , and $2 m_{-}$divides $m_{+},+1$.

4B2. $m_{-}$is a power of 2 , and $3 m_{-}=2\left(m_{+}+1\right)$.

Each condition corresponds to a different topological kind of example.

The above Theorem is proved by a detailed computation of the cohomology ring and the Stiefel-Whitney classes of the quotient spaces of the focal manifolds in $R P^{n+1}$ (which are both invariant under the antipodal involution of the sphere). A nice observation from those computations leads to [8], which proved that the situation 4B2 possibly occurs only when $m_{-}=2,4$ or 8 by Adams' Hopf invariants one theorem [2]. The known homogeneous examples show that $m_{-}=2$ or 4 do occur if the hypothesis 4B2 holds true. It is an open problem whether or not $m_{-}=8$ occurs. Note that $m_{+}=11$ if $m_{-}=8$ in the case $4 \mathrm{~B} 2$. We shall prove that $(8,11)$ cannot occur as the multiplicities and combining this with $[8]$ it follows that

Theorem A. In the case 4B2 the multiplicities $\left(m_{-}, m_{+}\right)$of an isoparametric hypersurface with four distinct principal curvatures must be either $(2,2)$ or $(4,5)$.

The proof of the Theorem above is based on the study of the topology of the focal manifolds which leads to the following Theorem B. We call two manifolds almost diffeomorphic if they are diffeomorphic up to connected sum with a certain homotopy sphere. Note that almost diffeomorphic implies homeomorphic if the dimension of the manifolds is not less than 4 by the well-known generalized Poincaré conjecture.

Without loss of generality, we may assume below that $m_{-} \leq m_{+}$. Since the isoparametric hypersurfaces with $g=4$ and $m_{-}=1$ have been classified up to isometry, let's restrict our attention to the case $m_{-}>1$ for the sake of simplicity.

Theorem B. The focal manifold $F_{-} \subset S^{n+1}$ is almost diffeomorphic to an $S^{m_{+}}$ bundle over $S^{m_{+}+m_{-}}$if one of the following conditions holds:

(1) $m_{+}>m_{-}$and $m_{+}=3,5,6$ or $7(\bmod 8)$.

(2) $m_{+}>2 m_{-}$and $m_{+}=0(\bmod 4)$;

where $m_{-} \neq 1$.

Thus the isoparametric hypersurface $M$ is homeomorphic to an iterated sphere bundle if the conditions of Theorem B hold. We remark that the same conclusion of Theorem B holds true for the focal manifolds of a proper Dupin hypersurface.

\section{Topology of the Focal MANifold $F_{-}$AND the Hypersurface $M$}

The Clifford isoparametric hypersurfaces are diffeomorphic to the iterated bundles and the focal manifolds are both sphere bundles over spheres [10]. Moreover, in many cases, the focal manifold $F_{-}$is diffeomorphic to the product of two spheres [10]. In this section, we are going to show that, in the topological category, some facts about the Clifford type isoparametric hypersurfaces and its focal manifolds can be extended generally as we stated in the above section.

Let $k \in\{-1,1\}=\{-,+\}$, and let $m_{k}$ be the multiplicities of the $\frac{g}{2}$-curvatures. Denote by $F_{k}$ the focal manifolds and by $M$ the isoparametric hypersurface. Note that $\operatorname{dim} F_{k}=2 m_{-k}+m_{k}$ and $\operatorname{dim} M=n=2 m_{-k}+2 m_{k} . M$ splits $S^{n+1}$ into two pieces which are both disc bundles over the focal manifold $F_{k}$ with fibre $D^{m_{k}+1}[7]$, 
[1]. By Morse theory the focal manifold $F_{k}$ is simply connected if $m_{-k}>1$ [7] and its homology groups are

$$
H^{*}\left(F_{k} ; Z\right) \cong \begin{cases}Z & \text { if } *=0, m_{-k}, m_{-k}+m_{k}, 2 m_{-k}+m_{k}, \\ 0 & \text { otherwise. }\end{cases}
$$

Thus $F_{k}$ is $\left(m_{-k}-1\right)$-connected and $\pi_{m_{-k}}\left(F_{k}\right) \cong Z$ by the Hurewicz Theorem. Denote by $\alpha$ a generator of $\pi_{m_{-k}}\left(F_{k}\right) \cong Z$; it can be represented by an embedded sphere $\alpha: S^{m_{-k}} \hookrightarrow F_{k}$. Now we are ready to begin our proof of Theorem B.

Proof of Theorem B. Let $\alpha$ be as above. If the condition (1) is satisfied, then the normal bundle $\gamma(\alpha)$ of $\alpha$ is trivial since $\widetilde{K O}\left(S^{m_{+}}\right)=0$. When $m_{+}=0(\bmod 4)$, the normal bundle $\gamma(\alpha)$ is trivial if and only if its $\frac{m_{+}}{4}$-th Pontryagin class vanishes. The restriction of the normal bundle of $F_{-}$in $S^{n+1}$ to $\alpha\left(S^{m_{+}}\right) \subset F_{-}$is classified by an element of $\pi_{m_{+-1}}\left(S O\left(m_{-}+1\right)\right)$. This group is a torsion group if the condition (2) is satisfied [3] and hence this restriction is stably trivial. Note that

$$
\left.\gamma(\alpha) \oplus \gamma\left(F_{-} \subset S^{n+1}\right)\right|_{S^{m_{+}}}
$$

is stably trivial and hence $\gamma(\alpha)$ is trivial. Thus the tubular neighborhood of $\alpha$ is diffeomorphic to $S^{m_{+}} \times D^{m_{+}+m_{-}}$.

By Alexander duality and Van-Kampen's theorem it is easy to see that the complement of the open tubular neighborhood of $\alpha\left(S^{m_{+}}\right)$in $F_{-}$is homotopy equivalent to $S^{m_{+}}$. By the standard handlebody theory it is also diffeomorphic to $S^{m_{+}} \times D^{m_{+}+m_{-}}$. Thus $F_{-}$is diffeomorphic to the glueing of the two copies of the handle $S^{m_{+}} \times D^{m_{+}+m_{-}}$along its boundary by a diffeomorphism $f \in$ Diff $S^{m_{+}} \times$ $S^{m_{+}+m_{-}-1}$. Notice that the diffeomorphism type of the result of the glueing depends only on the isotopy class of $f,[f] \in \pi_{0}$ Diff $S^{m_{+}} \times S^{m_{+}+m_{-}-1}$. Furthermore, this diffeomorphism type depends only on the class of $[f]$ in the quotient group of $\pi_{0}$ Diff $S^{m_{+}} \times S^{m_{+}+m_{-}-1}$ by the subgroup consisting of those diffeomorphisms extendable to $S^{m_{+}} \times D^{m_{+}+m_{-}}$(cf. [9]). As there is an orientation-reversing diffeomorphism of $S^{m_{+}} \times S^{m_{+}+m_{-}-1}$ extendable to $S^{m_{+}} \times D^{m_{+}+m_{-}}$, we can assume that the attaching diffeomorphism preserves the orientation.

Take A: $\pi_{0}$ Diff $S^{m_{+}} \times S^{m_{+}+m_{--1}} \rightarrow$ Aut $H_{*}\left(S^{m_{+}} \times S^{m_{+}+m_{-}-1}\right)$ to be the homomorphism given by $\mathbf{A}(f)=f_{*}$, the homomorphism induced by the diffeomorphism $f$. If the diffeomorphism $f$ preserves the orientation, by the duality theorem the automorphism $f_{*}$ is determined by its value on the $m_{+}$-th homology group. In our situation, where $m_{-}>1$, the $m_{+}$-th homology group is the integer group and a self isomorphism is either id or -id. Thus there always exists a diffeomorphism extendable to $S^{m_{+}} \times D^{m_{+}+m_{-}}$realizing the automorphism.

For each $\beta \in \pi_{m_{+}+m_{-}-1}\left(S O\left(m_{+}+1\right)\right)$ and $\delta \in \pi_{m_{+}}\left(S O\left(m_{+}+m_{-}\right)\right)$, there are two diffeomorphisms in ker $\mathbf{A}$ associated to $\beta$ and $\delta$ as follows:

$$
\begin{aligned}
& f_{\beta}: S^{m_{+}} \times S^{m_{+}+m_{-}-1} \rightarrow S^{m_{+}} \times S^{m_{+}+m_{-}-1} ; f_{\beta}(x, y)=(\beta(y) \cdot x, y), \\
& f_{\delta}: S^{m_{+}} \times S^{m_{+}+m_{-}-1} \rightarrow S^{m_{+}} \times S^{m_{+}+m_{-}-1} ; f_{\delta}(x, y)=(x, \delta(x) \cdot y) .
\end{aligned}
$$

When $m_{+}>m_{-}>1$, by [9] ker $\mathbf{A}$ is isomorphic to the direct sum

$$
\pi_{m_{+}}\left(S O\left(m_{+}+m_{-}\right)\right) \oplus \pi_{m_{+}+m_{--1}}\left(S O\left(m_{+}+1\right)\right) \oplus \Gamma^{2 m_{+}+m_{-}}
$$

and the former two terms given by a correspondence constructed above, where $\Gamma^{2 m_{+}+m_{-}}$is the group of $2 m_{+}+m_{-}$dimensional homotopy spheres, which can be 
identified naturally with the orientation-preserving diffeomorphism group $\pi_{0} S$ Diff $S^{2 m_{+}+m_{-}-1}$.

Obviously every element of ker $\mathbf{A}$ corresponding to the group

$$
\pi_{m_{+}}\left(S O\left(m_{+}+m_{-}\right)\right)
$$

by the above isomorphism can be extended to a diffeomorphism of $S^{m_{+}} \times D^{m_{+}+m_{-}}$. Thus $F_{-}$is diffeomorphic to the glueing of the two copies $S^{m_{+}} \times D^{m_{+}+m_{-}}$by a diffeomorphism of $S^{m_{+}} \times S^{m_{+}+m_{-}-1}$ corresponding to an element of

$$
\pi_{m_{+}+m_{-}-1}\left(S O\left(m_{+}+1\right)\right) \oplus \Gamma^{2 m_{+}+m_{-}}
$$

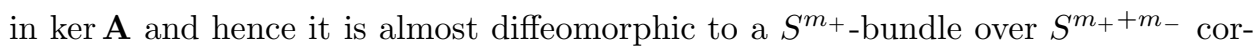
responding to a certain element in $\pi_{m_{+}+m_{-}-1}\left(S O\left(m_{+}+1\right)\right)$. This proves Theorem B.

\section{Proof of Theorem A}

By [8], in the case $4 \mathrm{~B} 2, m_{-}=2,4$ or 8 . For the reader's convenience, we include the simple argument here. By the computation of [1], in the case 4B2, the $m_{-}$-th Stiefel-Whitney class of the normal bundle $\gamma$ of $F_{+}$in the great sphere $S^{n+1}$ is nontrivial. Notice $F_{+}$is $\left(m_{-}-1\right)$-connected. Take an embedding $\alpha: S^{m_{-}} \rightarrow F_{+}$ to represent the $m_{-}$-dimensional generator of the homology group. This is possible by the Hurewicz Theorem. Thus the pullback bundle $\alpha^{*}(\gamma)$ is a bundle over the sphere $S^{m_{-}}$and has nontrivial $m_{-}$-th Stiefel-Whitney class. As $m_{-}$is even in the case $4 \mathrm{~B} 2$, this implies that $m_{-}=2,4$ or 8 by [2]. To prove our theorem, we only need to prove that $(8,11)$ cannot occur as the multiplicities of an isoparametric hypersurface with four distinct principal curvatures. Suppose it does occur; by Theorem B the focal manifold $F_{-}$is almost diffeomorphic to a $S^{11}$-bundle over $S^{19}$ which is given by an element $\xi \in \pi_{18}(S O(12))$. By the computation in [1] the normal bundle of $F_{+}$in the sphere $S^{39}$ has nontrivial 8-th Stiefel-Whitney class. Denote by $\gamma$ this normal bundle with $M(\gamma)$ its Thom complex and $U_{\gamma} \in H^{12}\left(M(\gamma) ; Z_{2}\right)$ its Thom class. By the definition of Stiefel-Whitney class, $S q^{8}\left(U_{\gamma}\right) \neq 0$. By the decomposition of the sphere into two ball bundles mentioned at the beginning of the last section, $M(\gamma)=D(\gamma) / S(\gamma) \simeq S^{39} / F_{-}$.

By the commutative diagram

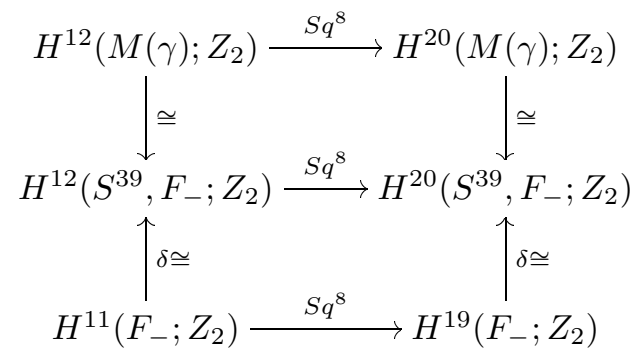

it follows that $S q^{8}: H^{11}\left(F_{-} ; Z_{2}\right) \rightarrow H^{19}\left(F_{-} ; Z_{2}\right)$ is nonzero and hence it is an isomorphism.

Now we are going to prove that, for any $S^{11}$-bundle over $S^{19}$, the action of $S q^{8}$ on the 11-th cohomology group vanishes and thus we reach a contradiction, which implies that the assumption that $(8,11)$ occurs as the multiplicities of an isoparametric hypersurface with four distinct principal curvatures is false and the proof follows. 
Now let us recall a standard fact in homotopy theory. Let $E$ be a bundle over $S^{m+1}$ with fiber $S^{k}$. Let $\chi \in \pi_{m}(S O(k+1))$ be the characteristic element for $E$. The sphere bundle $E$ has a cell decomposition $S^{k} \cup_{f} e^{m+1} \cup e^{k+m+1}$. We claim that the attaching map $f=p_{*}(\chi)$ up to homotopy, where $p: S O(k+1) \rightarrow S^{k}$ is the standard bundle projection and $p_{*}: \pi_{m}(S O(k+1)) \rightarrow \pi_{m}\left(S^{k}\right)$ its induced homomorphism. Note that $E=D^{m+1} \times S^{k} \cup_{g} D^{m+1} \times S^{k}$ where $g: \partial D^{m+1} \times S^{k} \rightarrow \partial D^{m+1} \times S^{k}$ is the glueing diffeomorphism, $g(x, y)=(x, \chi(x) \cdot y)$. Up to homotopy, we may think of the second handle in $E$ as $S^{k}$ and so $E=S^{k} \cup_{\pi_{2} \circ g} D^{m+1} \times S^{k}$ where $\pi_{2}$ is the projection to the second factor. $D^{m+1} \times S^{k}$ is a 3 -cell complex $S^{k} \cup e^{m+1} \cup e^{m+k+1}$. The restrictions of the glueing map $\pi_{2} \circ g$ to $\partial D^{m+1} \times(1,0, \ldots, 0)$ and $(1,0, \ldots, 0) \times S^{k}$ are exactly the map $p \circ \chi$ and the identity. Therefore $E$ has the homotopy type as claimed above. More details can be found in [11].

Applying this to our situation it follows that $F_{-}$has a cell decomposition with 20-dimensional skeleton the complex $S^{11} \cup_{\alpha} e^{19}$ up to homotopy, where $\alpha=p_{*}(\xi)$, $p_{*}: \pi_{18}(S O(12)) \rightarrow \pi_{18}\left(S^{11}\right)$. $S^{11}$ :

Consider the homotopy exact sequence for the fibre bundle $S O(11) \rightarrow S O(12) \rightarrow$

$$
\pi_{18}(S O(12)) \stackrel{p_{*}}{\longrightarrow} \pi_{18}\left(S^{11}\right) \rightarrow \pi_{17}(S O(11)) \stackrel{i_{*}}{\longrightarrow} \pi_{17}(S O(12)) .
$$

The group $\pi_{18}\left(S^{11}\right) \cong \frac{Z}{240}$ is just the 7 -th stable homotopy group of the sphere. If the kernel of $i_{*}: \pi_{17}(S O(11)) \rightarrow \pi_{17}(S O(12))$ has nontrivial 2-primary element, then $\operatorname{Im}\left(p_{*}\right)$ is generated by an even multiple of the generator $\eta \in \pi_{18}\left(S^{11}\right)$, thus $S q^{8}: H^{11}\left(S^{11} \cup_{\alpha} e^{19}\right) \rightarrow H^{19}\left(S^{11} \cup_{\alpha} e^{19}\right)$ vanishes and the proof follows.

Consider now the following diagram of exact sequences:

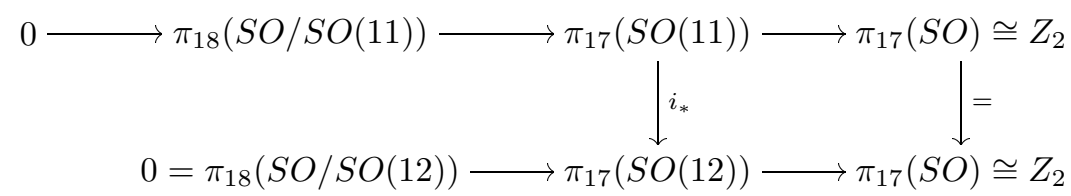

Then $\pi_{18}(S O / S O(12))=0$ and $\pi_{18}(S O / S O(11)) \cong Z_{2}$ by the table in [6]. The right commutative square is obvious. From the diagram it follows that the kernel of $i_{*}$ includes at least an order 2 element given by the image of $\pi_{18}(S O / S O(11))$ in $\pi_{17}(S O(11))$. Thus the proof follows.

\section{ACKNOWLEDGEMENT}

The present paper was written during the special year in 1995 on differential geometry in Nankai Institute of Mathematics. Some important new progress on the study of the multiplicity of isoparametric hypersurfaces has very recently been achieved independently in [12] and [13]. The author would like to thank Prof. S. S. Chern for his attention to this work and to thank Prof. G. Thorbergsson and Z. Tang for useful discussions. Finally I should like to thank the referee for his comments and suggestions.

\section{REFERENCES}

1. U. Abresch, Isoparametric hypersurfaces with four or six distinct principal curvatures, Math. Ann. 264 (1983), 283-302. MR 85g:53052b

2. J. F. Adams, On the non-existence of elements of Hopf invariant one, Ann. of Math. 72 (1960), 20-104. MR 25:4530 
3. A. Borel and F. Hirzebruch, Characteristic classes and Homogeneous spaces II, Amer. J. Math. 81 (1959), 315-382. MR 22:988

4. E. Cartan, Sur des familles remarquables d'hypersurfaces isoparametriques dans les espaces spheriques, Math. Z. 45 (1939), 335-367. MR 1:28f

5. A. Haefliger, Differentiable embeddings of $S^{n}$ in $S^{n+q}$ for $q>2$, Ann. of Math. 83 (1966), 402-436.

6. C. S. Hoo and M. E. Mahowald, Some homotopy groups of the Stiefel manifolds, Bull. Amer. Math. Soc. 71b (1965), 661-667. MR 31:1675

7. H. F. Münzner, Isoparametric hyperflächen in sphären I; II, Math. Ann. 251 (1980), 57-71; 256 (1981), 215-232. MR 82a:53058; MR 82m:53053

8. Z. Tang, Isoparametric hypersurfaces with four distinct principal curvatures, Chinese Science Bulletin 36 (1991), 1237-1240. MR 92m:53098

9. E. C. Turner, Diffeomorphisms of a product of spheres, Invent. Math. 8 (1969), 69-82. MR 40:3562

10. Q. Wang, On the topology of Clifford isoparametric hypersurfaces, J. Differential Geometry 27 (1988), 55-66. MR 89e:53093

11. I. M. James and J. H. C. Whitehead, The homotopy theory of sphere bundles over spheres (I), Proc. Lond. Math. Soc. (3) 4 (1954), 196-218. MR 15:892b

12. F. Fang, Multiplicities of principal curvatures of isoparametric hypersurfaces, Max-Planck Institut Preprint 96-80.

13. S. Stolz, Multiplicities of Dupin hypersurfaces, Max-Planck Institut Preprint 97-25.

Nankai Institute of Mathematics, Nankai University, Tianjin 300071, People's RePUBLIC OF CHINA

E-mail address: ffang@sun.nankai.edu.cn 